\title{
Nach der Novelle ist vor der Sanierung
}

Die Datenschutznovelle „II“ vom 14.08.2009, deren Neuregelungen überwiegend zum 01.09.2009 in Kraft getreten sind, bringt zahlreiche Änderungen in den Bestimmungen zum Direktmarketing und Datenhandel ( $\$ \S 28$ und 29 BDSG). Bisher war die Nutzung listenmäßig zusammengefasster ausgewählter personenbezogener Daten zu Zwecken der Werbung und des Adresshandels - im Rahmen der Bestimmungen des Wettbewerbsrechts - zulässig, sofern die schutzwürdigen Interessen des Betroffenen gewahrt wurden und der Betroffene der Nutzung nicht widersprochen hatte. In der nun gültigen Fassung des BDSG ist das Grundprinzip die Einwilligung des Betroffenen. Allerdings gibt es zahlreiche Ausnahmen von diesem Einwilligungsvorbehalt, die durch Querbezüge im Gesetzestext begrenzt wurden und sich nur mühsam in ihren wesentlichen Zügen erschließen lassen.

Tatsächlich hat die Klarheit insbesondere dieser Bestimmungen des novellierten BDSG im Verlauf des Gesetzgebungsprozesses stark gelitten. So sind beispielsweise Bestimmungen zur Einwilligung des Betroffenen in § 28 BDSG ergänzt worden nicht in $\S 4$ a (Einwilligung), wo man sie erwarten würde. $\S 28$ Abs. 3a fordert nun bei nicht schriftlich erklärten Einwilligungen, dass diese schriftlich bestätigt werden müssen - Ausnahme: die Erfüllung der Anforderungen an eine (elektronische) Einwilligung nach § 13 TMG. Auch verschwimmt im neuen BDSG die Trennlinie zum UWG: Die Regelung der als "unzumutbare Belästigung" geltenden und damit unzulässigen Werbemaßnahmen in § 7 UWG wird nun flankiert durch die Regelung der Zulässigkeit der Erhebung, Übermittlung und Nutzung bestimmter Adressdaten zum Zwecke der Werbung. Die Zulässigkeitsregelungen in UWG und BDSG fordern in zahlreichen Fällen nun zweierlei Arten von Einwilligungs- und Widerspruchsklauseln - die (grundsätzliche, datenschutzrechtliche) zur Erhebung personenbezogener (Adress-) Daten zum Zwecke der Werbung und eine zweite zur tatsächlichen werblichen Ansprache durch ein bestimmtes Unternehmen im konkreten Einzelfall.

So verständlich und ehrenwert die Motive des Gesetzgebers gewesen sein mögen, es muss ernsthaft bezweifelt werden, dass das Resultat der Neuregelung in der Praxis tatsächlich einen Fortschritt darstellt. Nicht nur die Formulierung angemessener und zugleich verständlicher Einwilligungs- und Widerspruchsklauseln wird einmal mehr zur Herausforderung. Auch deren praktische Umsetzung birgt zahlreiche Fallstricke. Allein die Differenzierung, ob ein werblich angesprochener Kunde nur über den verwendeten „Kanal“ (Post, Telefon, E-Mail, ...) oder generell von dem werbenden Unternehmen, vielleicht aber sogar überhaupt keine Werbung von einem (ihm unbekannten) Unternehmen mehr erhalten möchte, dürfte vielfach schwierig sein man stelle sich einen Call Center Mitarbeiter vor, der dies von einem verärgerten Gesprächspartner erfahren möchte. Das Resultat wird vermutlich ein Rückgang zulässiger personalisierter Werbung sein, denn viele Unternehmen werden „auf Nummer sicher" gehen und Widersprüche von Kunden eher weit auslegen. Damit wird der Anteil unzulässiger Werbung zunehmen - im subjektiven Empfinden der Betroffenen wird das kaum als ein Erfolg der Novellierung erscheinen.

Ähnliches gilt für weitere Bestimmungen des neuen BDSG, wie die Betrachtungen zum novellierten Gesetz von Jens Eckhardt zeigen. Noch keiner BDSG-Novelle zuvor ist es so eindrucksvoll gelungen zu belegen, dass eine Grundsanierung des Datenschutzrechts überfällig ist.

\section{Dirk Fox}

